\title{
Torsion as a Dark Matter Candidate from the Higgs Portal
}

\author{
Alexander S. Belyaev ${ }^{a, b, 1}$ Ilya L. Shapiro ${ }^{c, d, 2}$ Marc C. Thomas $^{a, b, 3}$ \\ School of Physics $\&$ Astronomy, University of S., Southampton SO17 1BJ, United Kingdom \\ Rutherford Appleton Laboratory,Science \& Technology Facilities Council (STFC),Chilton, Didcot. Oxon \\ OX11 OQX, United Kingdom \\ Departamento de Física - ICE, Universidade Federal de Juiz de Fora, Juiz de Fora, 36036-330, MG, \\ Brazil \\ Tomsk State Pedagogical University and Tomsk State University, Tomsk, 634041, Russia
}

\begin{abstract}
Torsion is a metric-independent component of gravitation, which may provide a more general geometry than the one taking place within general relativity. On the other hand torsion could lead to interesting phenomenology in both particle physics and cosmology. In the present work it is shown that a torsion field interacting with the SM Higgs doublet and having a negligible coupling to SM fermions is protected from decaying by a $Z_{2}$ symmetry, and therefore becomes a promising Dark Matter (DM) candidate. In order to check the consistency of this scenario we evaluate the DM relic density and explore direct DM detection and collider constraints on this model. It turns out that in the model when the Higgs boson is only partly responsible for the generation of torsion mass, there is a region of parameter space where torsion contributes $100 \%$ to the DM budget of the Universe. Furthermore, we show that the LHC currently has a limited sensitivity to the torsion parameter space via mono-jet signature and will be able to considerably improve its coverage of the torsion parameter space with the projected high luminosity.
\end{abstract}

KeYwords: Torsion, non-minimal parameters, Higgs, GUTs, Dark Matter, LHC

\footnotetext{
${ }^{1}$ e-mail: a.belyaev@soton.ac.uk

${ }^{2}$ e-mail: shapiro@fisica.ufjf.br

${ }^{3}$ e-mail: m.c.thomas@soton.ac.uk
} 


\section{Contents}

1 Introduction $\quad 1$

2 Interactions of torsion with fermions and scalars $\quad 2$

3 Torsion as a DM candidate $\quad 4$

4 Large hadron collider sensitivity to the torsion DM $\quad 9$

5 Conclusions $\quad 12$

\section{Introduction}

The existence of Dark Matter(DM) has been established beyond reasonable doubt at the cosmological scale, and is one of the main pieces of evidence of physics beyond the Standard Model(SM). There are several kinds of realistic extension of the SM which can explain the origin of DM. For instance, assuming some scenario of Grand Unification of electroweak and strong interactions at the UV scale, one naturally arrives at the concept of new weakly interacting particles which decouple from the baryonic sector in the Early Universe. This scenario serves as a theoretical grounding for models with weakly interacting massive particles - WIMPs, which appear in the majority of the theoretically motivated scenarios which predict a DM candidate, such as SUSY with R-parity [1, 2], Universal Extra Dimensions [3-6], Little Higgs [7-13] or Technicolor [14-16].

At the same time, one can consider different kinds of extensions of the SM of gravitational origin. One example, which is well-explored, is related to space-time torsion [17, 18]. An advantage of this kind of extension is that it follows from the unification of matter fields with gravity, including in the framework of (super)string theory. Indeed, superstring theory predicts the existence of torsion with a non-minimal coupling to scalar fields and fermions. As a result, the compactification of extra dimensions can in general give rise to a theory with a modified form of such non-minimal couplings in the low-energy limit.

At the GUT scale one has to use the field theory description instead of string theory, and therefore the first problem is to produce a consistent effective description of torsion and its interaction with matter fields. The last issue was addressed in the papers [19, 20] (see also [18, 21] for the case when torsion is a classical background for quantum matter fields). The main theoretical result was that the consistent formulation of such a theory requires a non-minimal interaction of the axial-vector component of torsion with fermions and also with scalar constituents of the theory. The non-minimal interactions are characterised by several free parameters, which can be defined only from experiments or observations. As far as these new parameters are introduced, the theory is consistently formulated at the semi-classical level.

The most difficult problem is related to formulating the theory of dynamical torsion which can provide a consistent effective quantum field theory description for the interaction with fermions of the SM. This issue was discussed in Refs. [22, 23], where it was shown that the consistent theory of antisymmetric torsion is described by the Proca-like action of axial vector fields. This results served 
as the basis for phenomenological studies (see also earlier work [24]), including an exploration of the LHC potential to probe torsion field [22, 23, 25].

In the present work we go beyond this framework and explore the phenomenological consequences of the non-minimal interaction between torsion and scalar fields. In particular we stress that in the case when the Higgs-fermion couplings vanish and torsion interacts with the SM particles only through the Higgs fields, torsion is protected from decaying by a $Z_{2}$ symmetry. Therefore in this case torsion becomes a good DM candidate. Torsion can also play the role of DM when its couplings to fermions are highly suppressed by the Planck mass, such that torsion's life time is of the order of the age of the Universe. In this paper we explore the potential of DM direct detection experiments as well as the LHC to probe the parameter space of this scenario.

The paper is organised as follows. In Sect. 2 we present a brief survey of the theory related to torsion interactions with fermions and scalars. Furthermore, a mechanism of generating relatively large non-minimal parameters for the torsion-Higgs interaction is sketched. In Sect. 3 we describe a torsion-based model of DM and in Sect. 4 discuss the possible potentials of the LHC, DM direct detection and Planck experiments to probe the scenario with torsion being a DM candidate. Finally, in Sect. 5 we draw our conclusions.

\section{Interactions of torsion with fermions and scalars}

Torsion $T_{\beta \gamma}^{\alpha}$ is a metric-independent tensor field which is defined as

$$
\Gamma_{\beta \gamma}^{\alpha}-\Gamma_{\gamma \beta}^{\alpha}=T_{\beta \gamma}^{\alpha} .
$$

One can split torsion into irreducible components

$$
T_{\alpha \beta \mu}=\frac{1}{3}\left(T_{\beta} g_{\alpha \mu}-T_{\mu} g_{\alpha \beta}\right)-\frac{1}{6} \varepsilon_{\alpha \beta \mu \nu} S^{\nu}+q_{\alpha \beta \mu},
$$

where the axial vector $S^{\nu}$ is dual to the completely antisymmetric part of the torsion tensor $S^{\nu}=\epsilon^{\alpha \beta \mu \nu} T_{\alpha \beta \mu}, T_{\alpha}=T_{\alpha \lambda}^{\lambda}$ is a vector trace of torsion and $q_{\alpha \beta \mu}$ is a tensor component.

The most general non-minimal action for a Dirac spinor coupled to torsion is

$$
S_{f}=\int \sqrt{-g}\left\{i \bar{\psi} \gamma^{\mu}\left(\nabla_{\mu}-i \eta_{1} \gamma^{5} S_{\mu}+i \eta_{2} T_{\mu}\right) \psi-m \bar{\psi} \psi\right\}
$$

where $\eta_{1}, \eta_{2}$ are non-minimal parameters and $\nabla_{\mu}$ is Riemannian covariant derivative, constructed without torsion. The minimal interaction corresponds to the values $\eta_{1}=-1 / 8$ and $\eta_{2}=0$. However, in the theory which includes scalar fields coupled to fermions via Yukawa interactions, the minimal theory is not renormalisable [19] and hence one has to introduce the non-minimal coupling of both fermions and scalars with the background torsion field. For instance, this can be seen from the renormalisation group equation for $\eta_{1}$,

$$
\mu \frac{d \eta_{1,2}}{d \mu}=\left(C_{1} h^{2}+\text { higher loop contributions }\right) \cdot \eta_{1,2},
$$

where $h$ is Yukawa coupling and the coefficient $C_{1}$ is model-dependent. It is clear that the minimal value $\eta_{1}=1 / 8$ is not stable under quantum corrections, hence one has to assume an arbitrary $\eta_{1}$. At the same time, $\eta_{2}=0$ does not lead to such a problem, therefore for the sake of simplicity one can restrict consideration by the purely antisymmetric torsion and the unique parameter $\eta_{1}$. 
In the scalar sector one meets the following non-minimal action:

$$
S_{0}=\int d^{4} \sqrt{-g}\left\{\nabla^{\mu} \varphi^{*} \nabla_{\mu} \varphi-m^{2}|\varphi|^{2}+\sum_{i=1}^{5} \xi_{i} P_{i}|\varphi|^{2}-f|\varphi|^{4}\right\},
$$

where $[19,20]$

$$
P_{1}=R, \quad P_{2}=\nabla_{\alpha} T^{\alpha}, \quad P_{3}=T_{\alpha} T^{\alpha}, \quad P_{4}=S_{\alpha} S^{\alpha}, \quad P_{5}=q_{\alpha \beta \gamma} q^{\alpha \beta \gamma} .
$$

In general, there can be up to five non-minimal parameters $\xi_{1 \ldots 5}$. However, if we restrict our attention to the antisymmetric torsion and flat space-time, the only one relevant parameter is $\xi_{4}$. The analysis of one-loop corrections shows that the non-minimal parameter $\xi_{4}$ and $\eta_{1}$ in the fermion sector are closely related, for instance the renormalisation group equation for $\xi_{4}$ has the general form

$$
\mu \frac{d \xi_{4}}{d \mu}=C_{2} h^{2} \eta_{1}^{2}+\left(C_{3} g^{2}+C_{4} g^{2}+C_{5} f\right) \xi_{4}+\text { higher loop contributions }
$$

with $g, h, f$ being gauge, Yukawa and scalar coupling constants (in the more realistic case, such as the SM, there will be several gauge and Yukawa-dependent terms) and model-dependent coefficients $C_{2, . .5}[19,21]$. Let us note that all that we have discussed until now corresponds to the case of a background torsion, which does not depend on the action of torsion. However, complete consideration should take into account the dynamics of torsion itself.

One can establish the action for the propagating torsion by requesting consistency of the effective low-energy quantum theory of torsion coupled to fermions and scalars, e.g., of the SM. Unitarity of the low-energy theory leads to a possible form of the torsion action $[22,23]$

$$
\mathcal{S}_{\text {tor }}^{T S-k i n}=\int d^{4} x\left\{-\frac{1}{4} S_{\mu \nu} S^{\mu \nu}+\frac{1}{2} M_{t s}^{2} S_{\mu} S^{\mu}\right\}
$$

where $S_{\mu \nu}=\partial_{\mu} S_{\nu}-\partial_{\nu} S_{\mu}$ and $M_{t s}$ is a torsion mass. Furthermore, one can show that quantum corrections preserve unitarity only if the relation

$$
\frac{M_{t s}^{2}}{\eta_{1}} \gg m_{f}^{2}
$$

is satisfied for all fermion fields with masses $m_{f}[22,23,26]$. In Ref. [27, 28] similar constraints in the theory with extra dimensions were discussed.

The consistency relations (2.8) concern torsion mass and non-minimal parameters of the fermiontorsion interaction. This condition can be satisfied either for a huge torsion mass or for a very weak fermion-torsion interaction. At this point we can conclude that the only torsion-related parameter which is not restricted is $\xi_{4}$ in Eq. (2.4). From the theoretical side there are no reasons to impose such a restriction. At the same time, there are no phenomenological constraints on $\xi_{4}$, because the present-day magnitude of the torsion field is extremely small [29]. Therefore it would be interesting to explore the physical consequences of different values of $\xi_{4}$, including of relatively large (compared to $\eta_{1}$ ) magnitudes, because they are not ruled out by the condition of theoretical consistency and, as we shall see below, may be phenomenologically fruitful.

Before we go to the phenomenological part in the next sections, let us present some additional theoretical arguments. One has to remember that $\xi_{4}$ is a free parameter and its value can be 
determined only by comparison to experimental or observational data. However, it is possible to sketch a situation when a relatively large value of $\xi_{4}$ may emerge ${ }^{1}$.

In the usual GUT models the SM Higgs couples not only to SM fermions, but also to other fields, which belong to the extension of the $S U(3)_{c} \times S U(2) \times U(1)$ gauge group. These fermions are supposed to have much greater masses compared to the SM particles. At the same time there is another possibility to decouple GUT particles from those of the SM. Namely, one can assume that the hidden UV sector of the GUT model is characterised by strong interactions, and that at an energy scale which is much lower than the GUT scale, these fields get confined into composite particles which have quantum numbers distinct from the original elementary particles. As a result they decouple from the SM particles. After such a decoupling only gravitational interactions are possible, hence these composite particles can constitute some part of the DM. In what follows we discuss the situation when the main part of the DM is composed by torsion itself. An advantage of this scheme is that torsion is a geometric field and, hence, may have very weak non-gravitational interaction to the SM particles.

An important aspect of the IR-confined GUT is that close to the decoupling scale there are typically very strong interactions and the quantum fields system is in the strongly non-perturbative regime. Then the specific GUT gauge, Yukawa and scalar couplings may be strong and one can observe an intensive running in Eq. (2.3) for the hidden fermions.

In this scenario the values of $\eta_{1}$ for the hidden fermions may greatly increase due to the intensive running. Since the masses of these fermions are supposed to be huge and, moreover, they are eventually confined into hidden composite particles, a possible violation of the criterion (2.8) does not put into danger the unitarity of the theory. But, as a consequence of interacting with the SM Higgs, Eq. (2.6) may produce a short-period but very intensive growth of the value of $\xi_{4}$ on the running from UV to IR. Therefore, within this scheme there is nothing wrong with assuming that at the low-energy of $\xi_{4}$ is "unnaturally" large. At the same time this process does not concern at all the SM fermions, hence the corresponding parameters $\eta_{1}$ remain very small. In the next sections we will discuss some phenomenological advantages of these assumptions.

\section{Torsion as a DM candidate}

Starting from this section, we shall use simplified notations $\xi_{4}=\xi$ and $\eta_{1}=\eta$. According to the previous considerations, the torsion-matter interaction has the form

$$
\mathcal{L}_{\text {tor }}^{\text {matter }}=\eta \sum_{i} \bar{\psi}_{i} \gamma^{\mu} \gamma^{5} \psi_{i} S_{\mu}
$$

where $\psi_{i}=e, \mu, \tau, \nu_{e}, \nu_{\mu}, \nu_{\tau}, u, c, t, d, s, b$

Furthermore, the Higgs portal looks as follows:

$$
\mathcal{L}_{\text {tor }}^{H}=\xi H^{2} S^{\mu} S_{\mu} .
$$

One can see that the Higgs mechanism generates the mass $M_{t s}^{2}=\xi v^{2}$ for the torsion $S^{\mu}$. The simplest scenario, in which this mechanism is the only source of the $S^{\mu}$-field mass, will be abbreviated hereafter as $T D M 1$, since in such a scenario the torsion mass, $M_{t s}$, is a function of only one parameter $\xi$. At the same time torsion can receive an additional mass contribution, which

\footnotetext{
${ }^{1}$ A Riemannian version of a similar arguments has been discussed recently in [30] in relation to the QFT-based mechanism for inflation.
} 
we denote as $M_{t s}^{\prime}$ which may come from the assumed symmetry breaking at the high energy scale. Therefore, one can write the general mass relation in the form

$$
M_{t s}^{2}=\xi v^{2}+M_{t s}^{\prime 2} .
$$

We will abbreviate this (more general) scenario as TDM2 since $M_{t s}$ in this case depends on two parameters. We have implemented both the TDM1 and TDM2 models into the CalcHEP package [31].

Because we are exploring the scenario where torsion is stable at the cosmological scale, its lifetime should be above 14 billion years, which translates into a torsion width below $10^{-42} \mathrm{GeV}$. This takes place for very small values of $\eta$, the non-minimal coupling of $S^{\mu}$ to fermions, the allowed region for which is indicated by coloured region in Fig 1 in the $\left(\eta-M_{t s}\right)$ plane. The white region is excluded because in these scenarios $\tau_{t s}<13.8$ billion years. One can see that this condition excludes values of $\eta>10^{-21}$. Hereafter we will be mainly interested in the torsion-scalar coupling. Therefore, since $\eta$ is so small, one can assume that it is zero, or that $\eta$ is below the aforementioned limit.

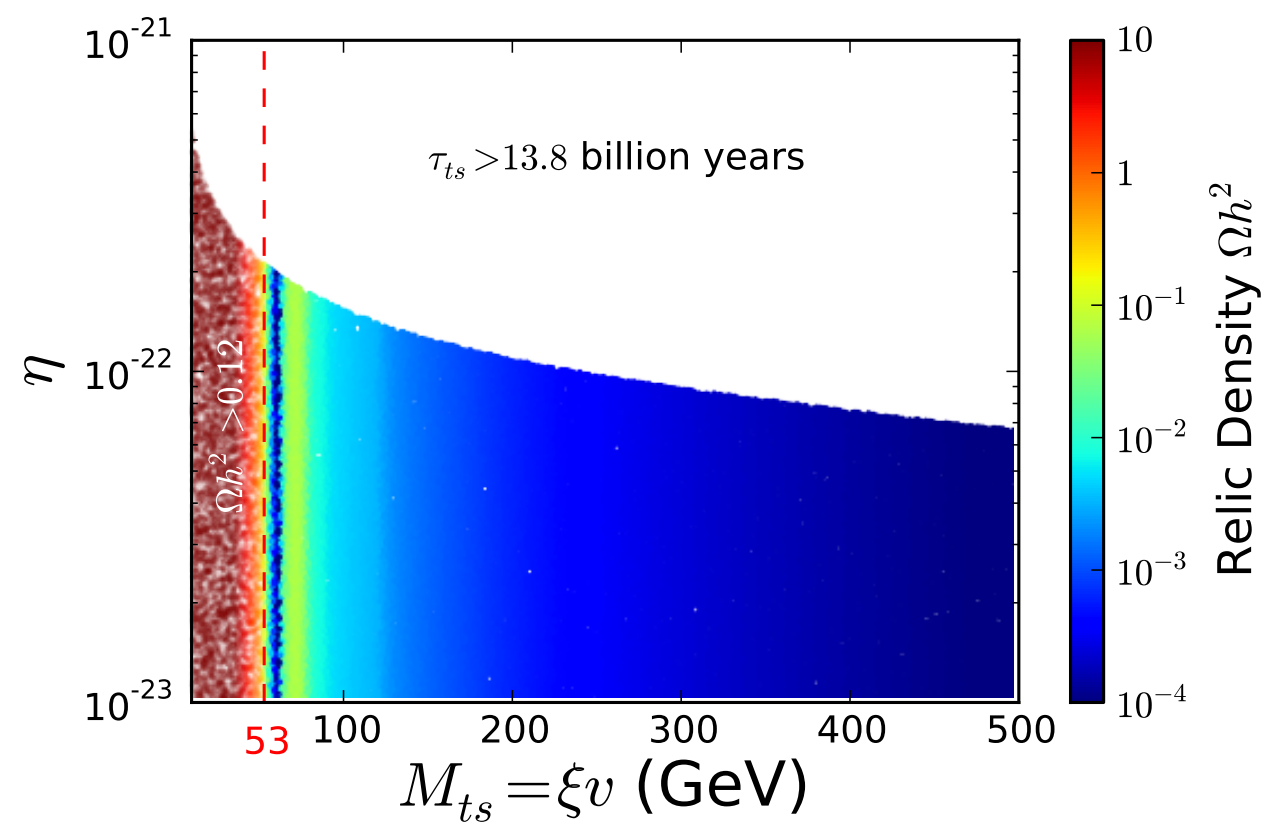

Figure 1. The upper limit on $\eta$ shown in the $\left(\eta-M_{t s}\right)$ plane. The coloured region is allowed and presents the torsion relic density in standard units of $\Omega h^{2}$. The white region above the coloured one is excluded because $\tau_{t s}<13.8$ billion years.

In this figure we also present a colour map of the torsion-DM relic density in standard units of $\Omega h^{2}$ which we evaluated using micrOMEGAs 4.2 .5 [32-34]. Precise measurements of the DM relic density from Planck [35, 36] (and previously from WMAP [37]),

$$
\Omega_{\mathrm{DM}}^{\text {Planck }} h^{2}=0.1184 \pm 0.0012,
$$

provide further constraints on the torsion parameter space. Taking this additional measurement into account, we have found that $M_{t s}$ below $53 \mathrm{GeV}$ is excluded in the TDM1 scenario. This 
is related to the fact that for such low $M_{t s}$ and respectively low values of $\xi=M_{t s} / v^{2}$, torsion DM in the early Universe annihilates into SM particles through an s-channel off-shell Higgs boson exchange. For $M_{t s}$ below $53 \mathrm{GeV}$, the suppression of this DM annihilation cross section leads to an overabundance of DM which is excluded by Planck data. The excluded parameter space is located to the left side of the dashed red line.

At the same time, once the mass of the torsion is close to $M_{H} / 2$, the effective annihilation through the on-shell Higgs boson resonance $S^{\mu} S^{\mu} \rightarrow H$ takes place. Since the cross section of this process is high, the relic density is about 2-3 orders of magnitude below the $\Omega_{\mathrm{DM}}^{\text {Planck }} h^{2}$. This region of parameter space, as well as other regions with low relic density are not necessarily excluded, since there could be additional (non-torsion) sources of DM contributing to the overall DM relic density.

We further constrain the torsion parameter space by using LHC bounds on the invisible Higgs boson decay,

$$
\operatorname{Br}(H \rightarrow \text { invisible })=\operatorname{Br}\left(H \rightarrow S^{\mu} S^{\mu}\right)<28 \%
$$

at the $95 \%$ confidence level (CL) [38]. In addition, we also check the spin-independent (SI) cross section of DM scattering off the nuclei relevant to DM direct detection (DD) searches and the respective current exclusion by the LUX [39] collaboration. We express the LUX sensitivity using the measure,

$$
R_{S I}=\frac{\tilde{\sigma}_{S I}}{\sigma_{S I}^{L U X}}=\frac{\sigma_{S I}}{\sigma_{S I}^{L U X}} \frac{\Omega h^{2}}{\Omega_{\mathrm{DM}}^{\text {Planck }} h^{2}}
$$

which contains the ratio of SI cross section for DM scattering on the nuclei and the LUX limit on the cross section multiplied by a DM relic density re-scaling factor $\frac{\Omega h^{2}}{\Omega_{\mathrm{DM}}^{\text {Panck }} h^{2}}$, to take into account the case when torsion relic density only partly contribute to the overall DM relic density of the Universe. Regions of torsion parameter space will be excluded if $R_{S I}>1$.

The effect of the Relic density, $\operatorname{Br}\left(H \rightarrow S^{\mu} S^{\mu}\right)$ and DM DD constraints are presented in Fig. 2. From this figure one can learn the following:

1) The region $M_{t s}<M_{H} / 2=62.5 \mathrm{GeV}$ is excluded by the constarint $\operatorname{Br}\left(H \rightarrow S^{\mu} S^{\mu}\right)$;

2) The regions $M_{t s} \lesssim 55 \mathrm{GeV}$ and $M_{H} / 2<M_{t s} \lesssim 145 \mathrm{GeV}$ are excluded by LUX DD constraints;

3) The region $M_{t s} \lesssim 53 \mathrm{GeV}$ is excluded by the relic density constraint, since in this region the torsion relic density is above the Planck limit.

Furthermore, the shape of the $R_{S I}$ line (which is correlated with the relic density behaviour) exhibits several representative peaks and troughs, namely:

a) the first sharp deep at $M_{H} / 2$ corresponds to the resonant DM annihilation through the Higgs boson which consequently leads to a sharp drop in the relic density;

b) the relic density sharply rises as soon as its mass is above $M_{H} / 2$ because it no longer annihilates via an exactly on-shell Higgs in the early Universe;

c) once $M_{t s}$ approaches $M_{W}$ and $M_{Z}$, the relic density drops again because the $S^{\mu} S^{\mu} \rightarrow W^{+} W^{-} / Z Z$ annihilation processes become available;

d) Finally, once $M_{t s}$ approaches $M_{H}$, one can see another step down in relic density due to the $S^{\mu} S^{\mu} \rightarrow H H$ processes. As $M_{t s}$ increases further beyond $M_{H}$, the $\xi$ coupling respectively increases leading to a further decrease in the relic density of the TDM1-model.

The combination and complementary of these constraints $\left(B r\left(H \rightarrow S^{\mu} S^{\mu}\right), \Omega h^{2}\right.$ and DM DD) allow scenarios where $M_{t s} \gtrsim 145 \mathrm{GeV}$, as well as a very narrow region with $M_{t s}$ just above 62.5 $\mathrm{GeV}$ (the Higgs resonance annihilation). In both allowed regions the relic density is one to two 


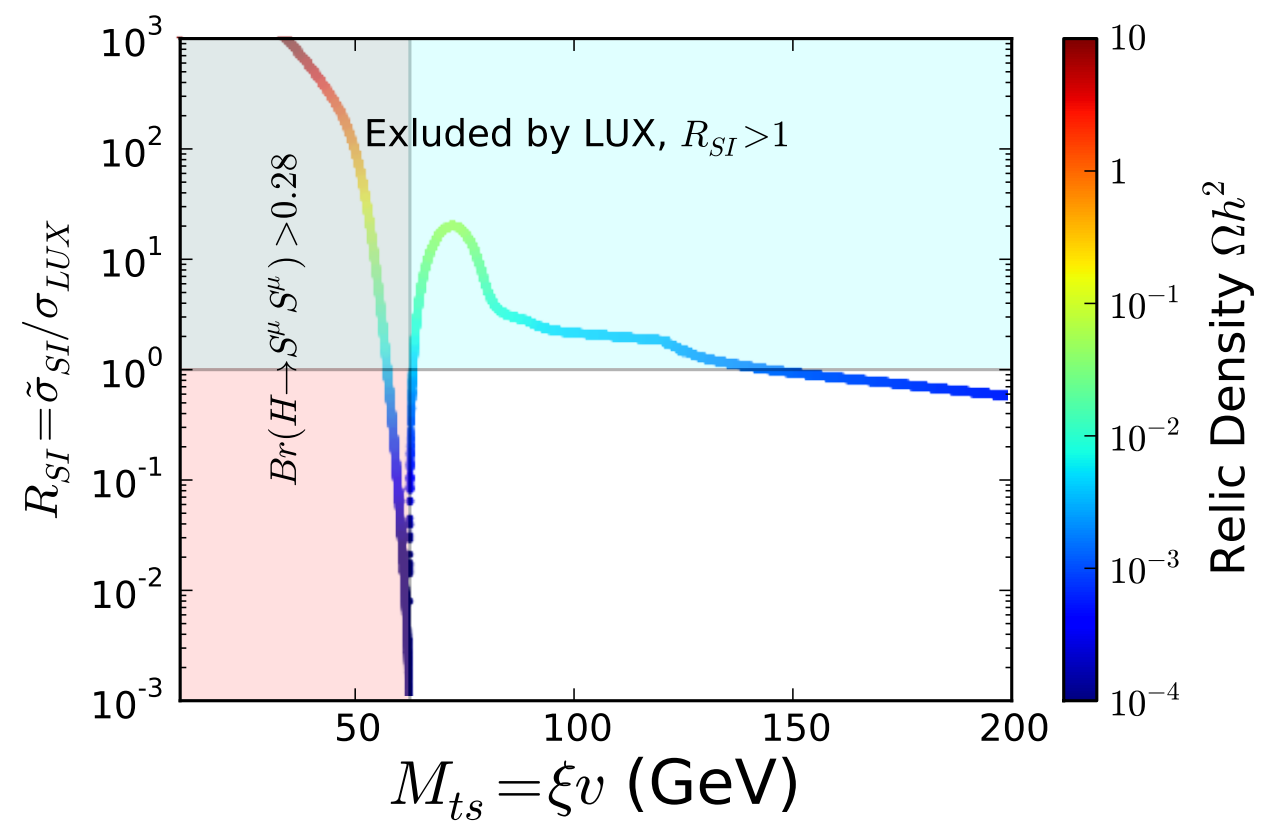

Figure 2. Relic density, $\operatorname{Br}\left(H \rightarrow S^{\mu} S^{\mu}\right)$ constraint and DM DD rates in the TDM1 scenario. The coloured line indicate the value of DM DD rate relative to the LUX constraint, $R_{S I}$ which excludes the model parameter space above $R_{S I}=1$ level (green-shaded area). At the same time the colour of this line indicate the value of the relic density, $\Omega h^{2}$ which exceeds Planck constraint at the $M_{t s} \simeq 53$, which therefore excludes parameter space below this value. In the pink area, the torsion parameter space is excluded by constraints on $\operatorname{Br}\left(H \rightarrow S^{\mu} S^{\mu}\right)$ from the Higgs invisible decay searches.

orders of magnitude below $\Omega_{\mathrm{DM}}^{\mathrm{Planck}} h^{2}$, and therefore in the one-parametric TDM1 scenario torsion never contributes $100 \%$ to the total budget of DM.

Let us now consider TDM2 scenario, corresponding to more general case when torsion acquires an additional mass, $M_{t s}^{\prime}$, from symmetry breaking(s) at a higher energy scale, such as in a GUT. Then one can consider this mass as an additional parameter, which would allow torsion to make up $100 \%$ of the DM budget. In Fig. 3, we present the TDM2 parameter space in the $\xi-M_{t s}$ plane, following application of constraints from $\operatorname{Br}\left(H \rightarrow S^{\mu} S^{\mu}\right), \Omega h^{2}$ and DM DD. In this case

$$
M_{t s}=\sqrt{\xi v^{2}+M_{t s}^{\prime 2}}
$$

and $\xi$ and $M_{t s}$ can be regarded as independent parameters.

Fig. 3(a) presents the colour map of $\Omega h^{2}$ in the unconstrained parameter space, Fig. 3(b) demonstrates the effect of the $\operatorname{Br}\left(H \rightarrow S^{\mu} S^{\mu}\right)<0.28$ constraint and Fig. 3(c) shows the effect of the additional application of $R_{S I}$ constraint. Finally, Fig. 3(d) presents the parameter space after applying an upper bound on $\Omega h^{2}$. The coloured parameter space indicates the parameter space which survives the respective constraints. The upper edge of the coloured space indicated by the black contour corresponds to the TDM1 scenario. One can see that the allowed $M_{t s}$ mass range looks very similar to the TDM1 case. However, contrary to the TDM1 scenario, for any mass $M_{t s}$ one can find in the TDM2 parameter space value(s) of the $\xi$ parameter for which torsion contributes $100 \%$ to the DM relic density budget. This TDM2 scenario, where all constraints are 


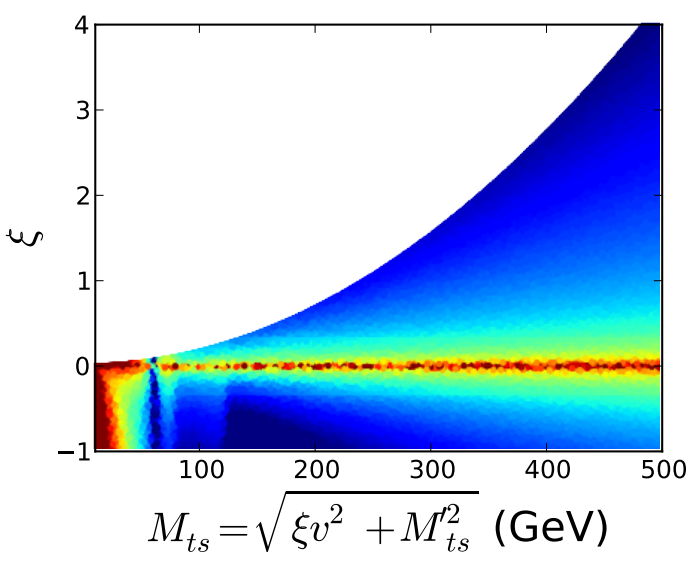

(a)

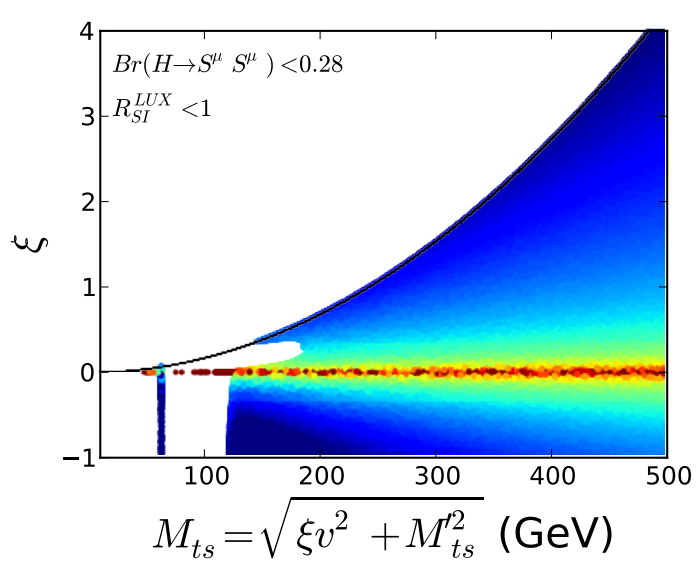

(c)

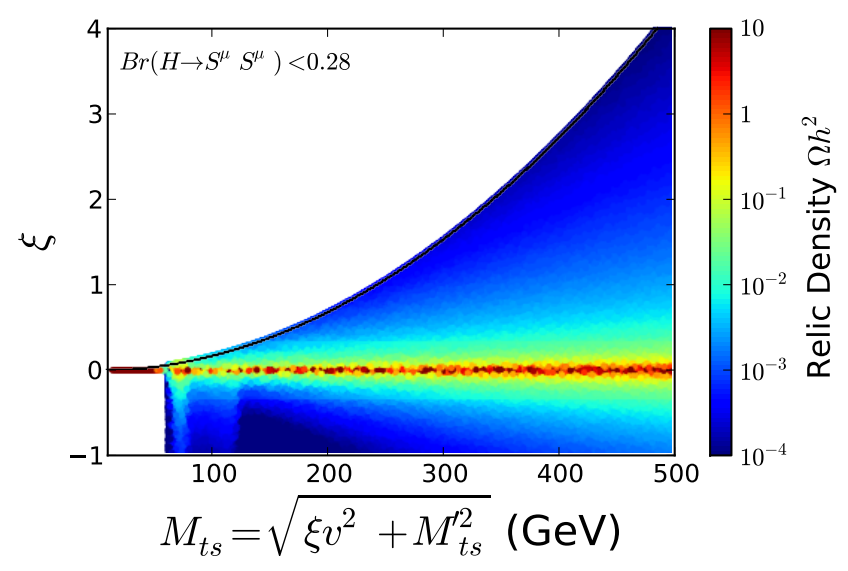

(b)

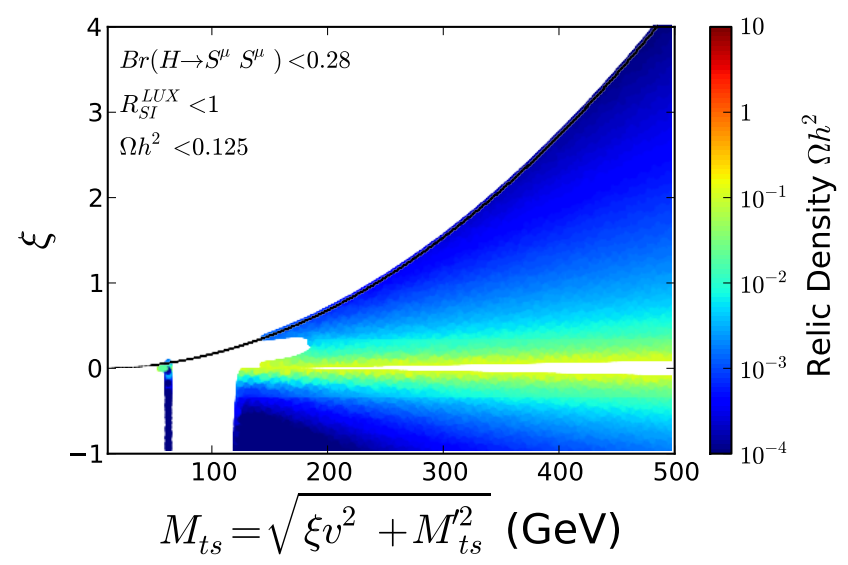

(d)

Figure 3. The $\Omega h^{2}$ colour map in the $\xi-M_{t s}$ parameter space of the TDM2 scenario which survives the respective constraints: a) the unconstrained parameter space; b) $B r\left(H \rightarrow S^{\mu} S^{\mu}\right)<0.28$ constraint applied; c) $R_{S I}$ constraint applied in addition; d) upper bound on $\Omega h^{2}$ applied on the top of the previous constraints. The white colour indicates the excluded region, while the coloured area is allowed by the set of constraints indicated for each frame.

satisfied, including that the entire relic density is explained with torsion (including the lower cut on $\Omega h^{2}$ not present in Fig. 3(d)) is denoted by the green-coloured region in Fig. 4. One can see that even for $M_{t s} \simeq M_{H} / 2$ one can have $\Omega h^{2} \simeq 0.112$, i.e., it is possible to find scenario when torsion is the sole Dark Matter. This scenario can be realised for small values of $\xi(\simeq 0.01)$ and the values of the GUT contribution to torsion mass, $M^{\prime}{ }_{t s}$, which are close to $M_{H} / 2$. One can also see that when $M_{t s} \gtrsim 135 \mathrm{GeV}$, there are regions of parameter space, represented by the green bands symmetric about the $\xi=0$ line, where torsion provides $100 \%$ of the relic density budget. 


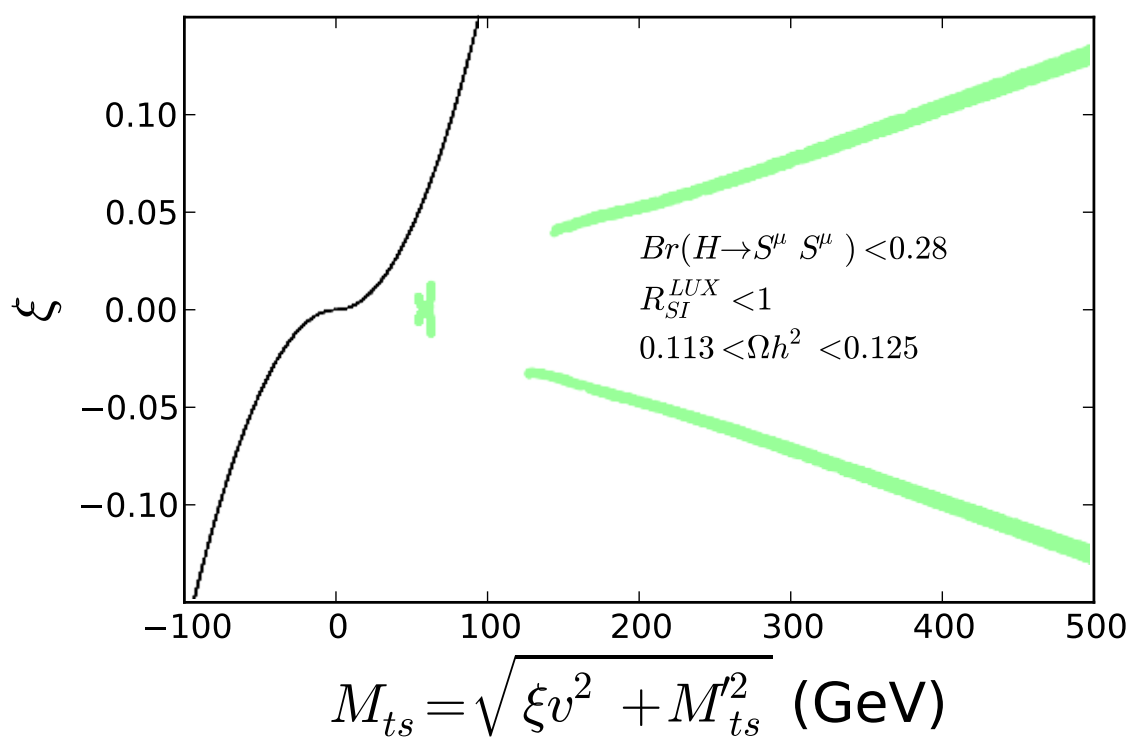

Figure 4. The TDM2 parameter space which survives $\operatorname{Br}\left(H \rightarrow S^{\mu} S^{\mu}\right)<0.28, R_{S I}<1$ and $0.113<$ $\Omega h^{2}<0.125$ cuts. The allowed region is shown in green.

\section{Large hadron collider sensitivity to the torsion DM}

In this section we find the current and project limits from the LHC on the torsion DM parameter space. Torsion, being a DM, gives rise mono-jet signatures at the LHC, $p p \rightarrow S^{\mu} S^{\mu}$ jet, when a pair of torsion particles is recoiled against the hard quark or gluon jet coming from the initial state radiation. Feynman diagrams for this process are presented in Fig. 5. The relevant parameter space
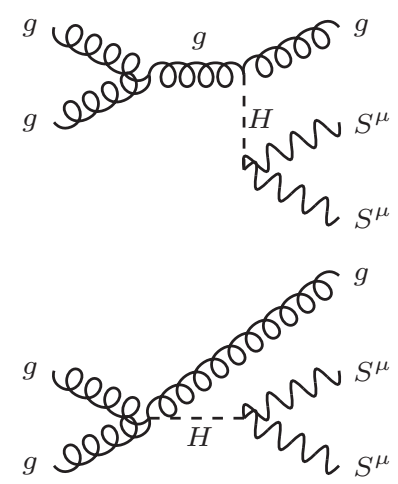
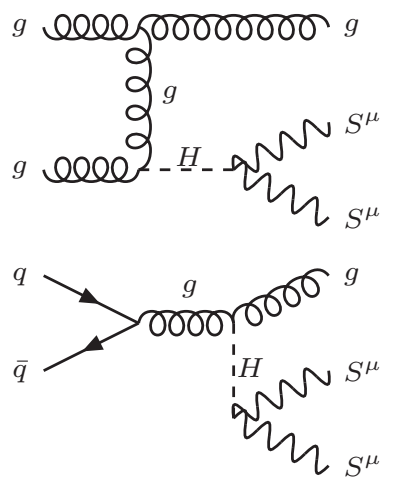
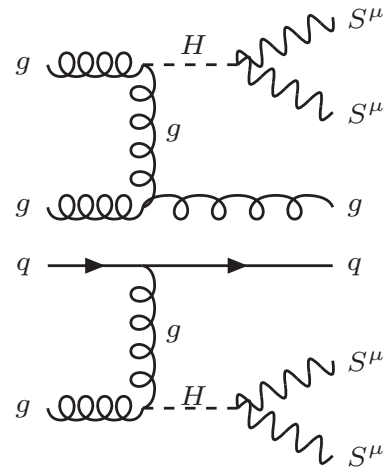

Figure 5. Feynman diagrams for the mono-jet signature $p p \rightarrow S^{\mu} S^{\mu}$ jet of the torsion pair production at the LHC.

for the model is eventually two dimensional, consisting of the $\xi$ and $M_{t s}$ parameters. There is an upper limit on the value of $\xi=\xi_{\max +}=M_{t s}^{2} / v e v^{2}$, coming from the $M_{t s}^{\prime}>0$ requirement. There is no limit on the absolute value of $\xi$ for $\xi<0$, except the perturbativity one.

In Fig. 6 we present the cross sections for the torsion mono-jet signature versus $M_{t s}$ at LHC@13 $\mathrm{TeV}$ with a $\boldsymbol{E}_{\mathrm{T}}^{\text {miss }}>100 \mathrm{GeV}$ cut, for two values of $\xi: \xi=0.01$ and $\xi=\xi_{\max +}$. In this evaluation we have used the QCD renormalisation and factorisation scales, $Q$ equal to the transverse momentum 


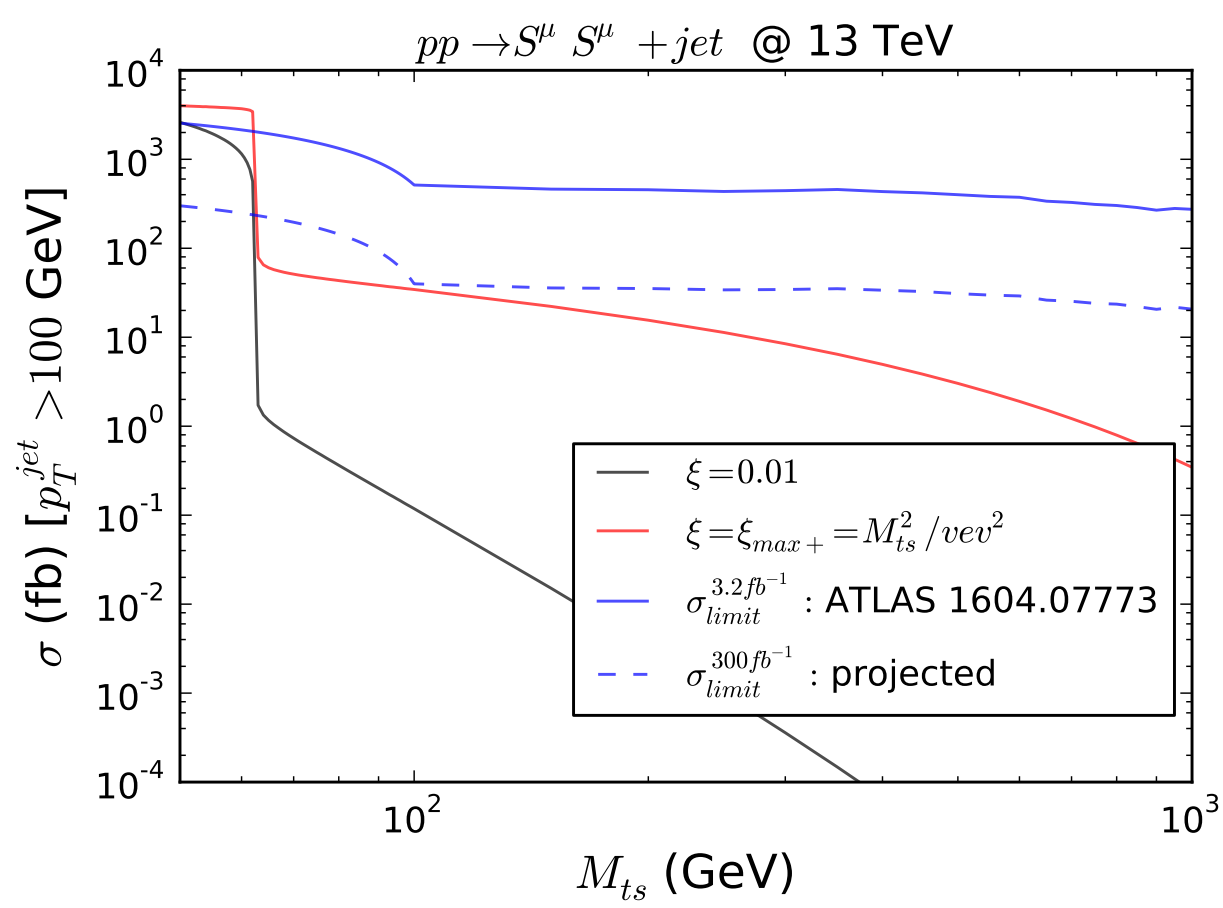

Figure 6. Cross sections for the torsion mono-jet signature versus $M_{t s}$ at LHC@13 TeV with the cut $\boldsymbol{E}_{\mathrm{T}}^{\text {miss }}>100 \mathrm{GeV}$ for two values of $\xi: \xi=0.01$ (solid black) and $\xi=\xi_{\text {max+ }}$ (solid red) as well as LHC limits obtained using an interpretation of ATLAS mono-jet analysis of Ref. [40] for $3.2 \mathrm{fb}^{-1}$ (solid blue) and projected $300 \mathrm{fb}^{-1}$ (dashed blue) integrated luminosities.

of the pair of DM particles i.e. missing transverse momentum, $\boldsymbol{E}_{\mathrm{T}}^{\mathrm{miss}}$, while the parton density function, PDF was chosen to be NNPDF23LO (as_0119_qed) PDF set [41]. In the same Figure we present LHC limits obtained using an interpretation of the ATLAS mono-jet analysis of Ref. [40] for $3.2 \mathrm{fb}^{-1}$ integrated luminosity. For our analysis we performed parton level simulation with CalcHEP, followed by PYTHIA8 [42] and DeLPHEs 3 [43] to simulate hadronisation and patron showering, and for fast detector simulation respectively. The detector level analysis was performed using CheckMATE v2 [44]. In Fig. 6 we also show the the limits on the cross section for the projected luminosities of $300 \mathrm{fb}^{-1}$ for these ATLAS analysis. Our projection is based on the assumptions that the number of BG events scales with the luminosity and that the uncertainty on the BG scales as the square root of the luminosity. However, we set the lower limit for the BG uncertainty to be $1 \%$ of the BG. This choice of $1 \%$ for the limit on BG uncertainty is based on the post-fit numbers with respective BG error provided by ATLAS and CMS for $\boldsymbol{E}_{\mathrm{T}}^{\text {miss }}$ bins with high statistics, see e.g. $[40,45]$ together with additional materials provided at http://cms-results. web.cern.ch/cms-results/public-results/preliminary-results/EXO-16-013/\#AddFig.

From Fig. 6 one can see that even for projected $300 \mathrm{fb}^{-1}$ integrated luminosity the LHC is not sensitive to the torsion parameter space for $M_{t s}>M_{H} / 2$ and positive values of $\xi$ coupling. Moreover, data for $3.2 \mathrm{fb}^{-1}$ does not set any limits on $M_{t s}$ for $\xi>0$. In Fig. 7 we present the LHC limits in the $\xi-M_{t s}$ plane which we obtained using limits presented in Fig. 6. The upper panel of the figure demonstrates that the current LHC data exclude the $M_{t s} \simeq M H / 2$ parameter space only for negative values of $\xi$ below -0.35 , indicated by dark pink colour. The bottom panel of Fig. 7 presents the region of the $\xi-M_{t s}$ plane zoomed in around $M_{t s} \simeq M_{H} / 2$ demonstrating that at 
$300 \mathrm{fb}^{-1}$ the LHC can probe $M_{t s}<M_{H} / 2$ masses for $|\xi|$ as low as about $2 \times 10^{-3}$, as indicated by the light-pink colour. From the top panel one can also see that at $300 \mathrm{fb}^{-1}$, the LHC can probe the $M_{t s}>M_{H}$ region for $\xi \lesssim-0.2$ as well, as is also shown by the shaded light-pink colour.
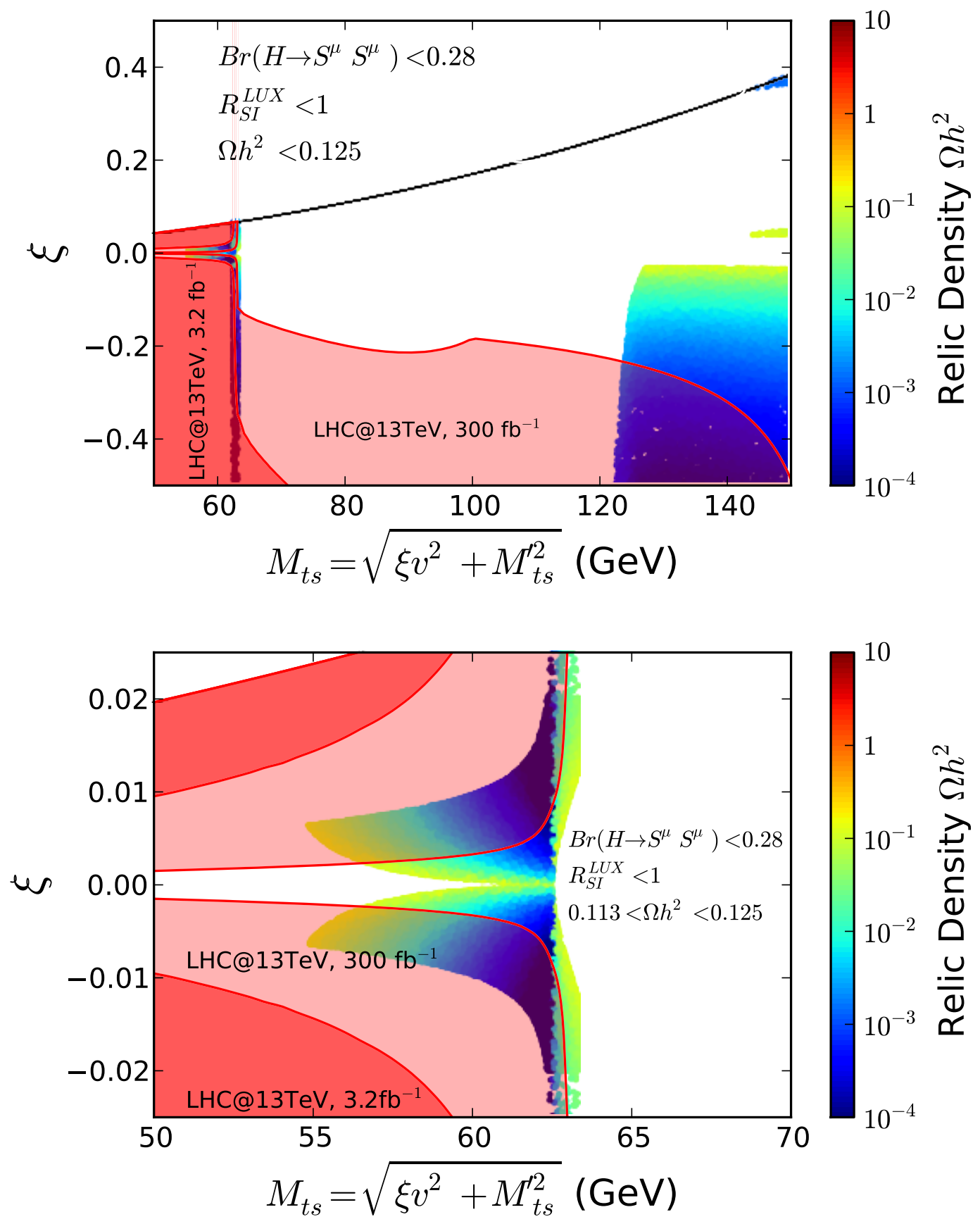

Figure 7. The LHC limits in $\xi-M_{t s}$ pane obtained using limits presented in Fig. 6. The bottom panel presents the region of $\xi-M_{t s}$ pane zoomed around $M_{H} / 2$. 


\section{Conclusions}

The standard argument in favour of torsion is that it provides a geometric counterpart for the spin of matter, which looks a natural thing in the microscopic physics. The consistency of quantum theory of matter fields on a torsion background requires torsion to be non-minimally coupled to both fermions of scalars of the SM. On the other hand torsion leads to interesting particle physics phenomenology, and also has important cosmological consequences (see, e.g., [46] for the review). We have considered a new application which combines naturally these two aspects. The theory of a torsion field interacting with the SM Higgs doublet and having negligible coupling to SM fermions is protected from decaying by a $Z_{2}$ symmetry and therefore becomes a promising DM candidate.

We have explored this possibility and checked the viability of this scenario in the light of DM relic density, direct detection and collider constraints. In the simplest $T D M 1$ scenario with just one parameter $\xi$ of the torsion-Higgs coupling we have found that torsion can not explain $100 \%$ of the DM relic density because of the tension with DM DD constraints. However in the two parametric $\xi-M_{t s} T D M 2$ scenario, when the Higgs boson is only partly responsible for generation of the torsion mass, there is a region of the parameter space where torsion is the sole DM. In this scenario the mass relation (3.7) which appears naturally within the GUT models provides an additional degree of freedom which allows the TDM2 to survive DM DD constraints.

We have also found that the LHC, while not being sensitive to TDM2 parameter space, anyway can further constrain it with the projected luminosity. For example, we have demonstrated that at $300 \mathrm{fb}^{-1}$ the LHC can probe masses $M_{t s}<M_{H} / 2$ for $|\xi|$ as low as about $2 \times 10^{-3}$, including the parameter space where torsion contributes $100 \%$ to the DM budget of the Universe. On the other hand at $300 \mathrm{fb}^{-1}$, the LHC can also probe the $M_{t s}>M_{H}$ scenario for $\xi \lesssim-0.2$, although in this case torsion's contribution to the DM relic density is at about the percent level or below.

\section{Acknowledgements.}

The present work was started during a short-term visit of A.B. to Juiz de Fora. The authors are very grateful to $\mathrm{CNPq}$ for supporting this visit. A.B. acknowledges partial support from the STFC grant number ST/L000296/1, NExT Institute and partial funding by a Soton-FAPESP grant. A.B. also thanks the Royal Society Leverhulme Trust Senior Research Fellowship LT140094. A.B. and M.T. would like to thank FAPESP grant 2011/11973-4 for funding their visit to ICTP-SAIFR where part of this work was completed. I.Sh. thanks CNPq, FAPEMIG and ICTP for partial support.

\section{References}

[1] H. Goldberg. Constraint on the Photino Mass from Cosmology. Phys. Rev. Lett., 50:1419, 1983. [Erratum: Phys. Rev. Lett.103,099905(2009)].

[2] John R. Ellis, J. S. Hagelin, Dimitri V. Nanopoulos, Keith A. Olive, and M. Srednicki. Supersymmetric Relics from the Big Bang. Nucl. Phys., B238:453-476, 1984.

[3] Ignatios Antoniadis. A Possible new dimension at a few TeV. Phys.Lett., B246:377-384, 1990.

[4] Thomas Appelquist, Hsin-Chia Cheng, and Bogdan A. Dobrescu. Bounds on universal extra dimensions. Phys.Rev., D64:035002, 2001.

[5] Geraldine Servant and Timothy M. P. Tait. Is the lightest Kaluza-Klein particle a viable dark matter candidate? Nucl. Phys., B650:391-419, 2003. 
[6] Csaba Csaki, Christophe Grojean, Jay Hubisz, Yuri Shirman, and John Terning. Fermions on an interval: Quark and lepton masses without a Higgs. Phys.Rev., D70:015012, 2004.

[7] N. Arkani-Hamed, A.G. Cohen, E. Katz, A.E. Nelson, T. Gregoire, et al. The Minimal moose for a little Higgs. JHEP, 0208:021, 2002.

[8] Hsin-Chia Cheng and Ian Low. TeV symmetry and the little hierarchy problem. JHEP, 09:051, 2003.

[9] Hsin-Chia Cheng and Ian Low. Little hierarchy, little Higgses, and a little symmetry. JHEP, 08:061, 2004.

[10] Ian Low. T parity and the littlest Higgs. JHEP, 10:067, 2004.

[11] Jay Hubisz and Patrick Meade. Phenomenology of the littlest Higgs with T-parity. Phys. Rev., D71:035016, 2005.

[12] Hsin-Chia Cheng, Ian Low, and Lian-Tao Wang. Top partners in little Higgs theories with T-parity. Phys. Rev., D74:055001, 2006.

[13] Jay Hubisz, Patrick Meade, Andrew Noble, and Maxim Perelstein. Electroweak precision constraints on the littlest Higgs model with T parity. JHEP, 01:135, 2006.

[14] S. Nussinov. Technocosmology: could a technibaryon excess provide a 'natural' missing mass candidate? Phys. Lett., B165:55-58, 1985.

[15] Stephen M. Barr, R. Sekhar Chivukula, and Edward Farhi. Electroweak Fermion Number Violation and the Production of Stable Particles in the Early Universe. Phys. Lett., B241:387-391, 1990.

[16] Sven Bjarke Gudnason, Chris Kouvaris, and Francesco Sannino. Towards working technicolor: Effective theories and dark matter. Phys. Rev., D73:115003, 2006.

[17] F. W. Hehl, P. Von Der Heyde, G. D. Kerlick, and J. M. Nester. General Relativity with Spin and Torsion: Foundations and Prospects. Rev. Mod. Phys., 48:393-416, 1976.

[18] I. L. Shapiro. Physical aspects of the space-time torsion. Phys. Rept., 357:113, 2002.

[19] I. L. Buchbinder and I. L. Shapiro. On the renormalization of models of quantum field theory in an external gravitational field with torsion. Phys. Lett., B151:263-266, 1985.

[20] I. L. Buchbinder and I. L. Shapiro. On the renormalization group equations in curved space-time with torsion. Class. Quant. Grav., 7:1197-1206, 1990.

[21] I. L. Buchbinder, S. D. Odintsov, and I. L. Shapiro. Effective action in quantum gravity. 1992.

[22] A. S. Belyaev and Ilya L. Shapiro. The Action for the (propagating) torsion and the limits on the torsion parameters from present experimental data. Phys. Lett., B425:246-254, 1998.

[23] A. S. Belyaev and I. L. Shapiro. Torsion action and its possible observables. Nucl. Phys., B543:20-46, 1999.

[24] S.M. Carroll and G.B. Field. Consequences of propagating torsion in connection dynamic theories of gravity. Phys. Rev., D50:3867-3873, 1994.

[25] A. S. Belyaev, I. L. Shapiro, and M. A. B. do Vale. Torsion phenomenology at the LHC. Phys. Rev., D75:034014, 2007.

[26] G. de Berredo-Peixoto, J. A. Helayel-Neto, and I. L. Shapiro. On the consistency of a fermion torsion effective theory. JHEP, 02:003, 2000.

[27] L.N. Chang, O. Lebedev, W. Loinaz, and Tatsu Takeuchi. Universal torsion induced interaction from large extra dimensions. Phys. Rev. Lett., 85:3765-3768, 2000. 
[28] O. Lebedev. Torsion constraints in the Randall-Sundrum scenario. Phys. Rev., D65:124008, 2002.

[29] A.V. Kostelecky and J.D. Tasson. Matter-gravity couplings and Lorentz violation. Phys. Rev., D83:016013, 2011.

[30] T. de Paula Netto, A.M. Pelinson, I.L. Shapiro, and A.A. Starobinsky. From stable to unstable anomaly-induced inflation. 2015.

[31] A. Belyaev, Christensen N. D., and A. Pukhov. CalcHEP 3.4 for collider physics within and beyond the Standard Model. Comput. Phys. Commun., 184:1729, 2013.

[32] G. Belanger, F. Boudjema, A. Pukhov, and A. Semenov. micrOMEGAs_3: A program for calculating dark matter observables. Comput. Phys. Commun., 185:960-985, 2014.

[33] G. Belanger, F. Boudjema, A. Pukhov, and A. Semenov. MicrOMEGAs 2.0: A Program to calculate the relic density of dark matter in a generic model. Comput. Phys. Commun., 176:367-382, 2007.

[34] G. Belanger, F. Boudjema, P. Brun, A. Pukhov, S. Rosier-Lees, P. Salati, and A. Semenov. Indirect search for dark matter with micrOMEGAs2.4. Comput. Phys. Commun., 182:842-856, 2011.

[35] P. A. R. Ade et al. Planck 2013 results. XVI. Cosmological parameters. Astron. Astrophys., 571:A16, 2014.

[36] P.A.R. Ade et al. Planck 2015 results. XIII. Cosmological parameters. 2015.

[37] G. Hinshaw et al. Nine-Year Wilkinson Microwave Anisotropy Probe (WMAP) Observations: Cosmological Parameter Results. Astrophys. J. Suppl., 208:19, 2013.

[38] G. Aad et al. Search for invisible decays of a Higgs boson using vector-boson fusion in $p p$ collisions at $\sqrt{s}=8 \mathrm{TeV}$ with the ATLAS detector. 2015.

[39] D. S. Akerib et al. First results from the LUX dark matter experiment at the Sanford Underground Research Facility. Phys. Rev. Lett., 112:091303, 2014.

[40] Morad Aaboud et al. Search for new phenomena in final states with an energetic jet and large missing transverse momentum in $p p$ collisions at $\sqrt{s}=13 \mathrm{TeV}$ using the ATLAS detector. 2016 .

[41] Richard D. Ball et al. Parton distributions with LHC data. Nucl. Phys., B867:244-289, 2013.

[42] Torbjorn Sjostrand, Stephen Mrenna, and Peter Z. Skands. A Brief Introduction to PYTHIA 8.1. Comput. Phys. Commun., 178:852-867, 2008.

[43] J. de Favereau et al. DELPHES 3, A modular framework for fast simulation of a generic collider experiment. JHEP, 1402:057, 2014.

[44] Manuel Drees, Herbi Dreiner, Daniel Schmeier, Jamie Tattersall, and Jong Soo Kim. CheckMATE: Confronting your Favourite New Physics Model with LHC Data. Comput. Phys. Commun., 187:227-265, 2014.

[45] CMS Collaboration. Search for dark matter production in association with jets, or hadronically decaying $\mathrm{W}$ or $\mathrm{Z}$ boson at $\sqrt{s}=13 \mathrm{TeV}$. CMS-PAS-EXO-16-013, 2016.

[46] Salvatore Capozziello and Mariafelicia De Laurentis. Extended Theories of Gravity. Phys. Rept., 509:167-321, 2011. 